\title{
Association of dialysis-requiring acute kidney injury with 90-day prognosis in patients with coronary artery disease and advanced kidney disease after coronary angiography
}

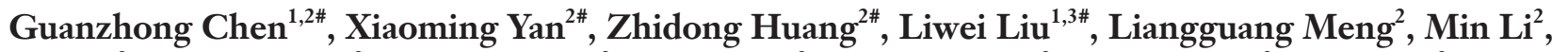 \\ Jin Liu ${ }^{2}$, Shiqun Chen ${ }^{2}$, Huanqiang $\mathrm{Li}^{2}$, Ziling Mai ${ }^{2}$, Enzhao Chen ${ }^{2}$, Disheng Lai ${ }^{2}$, Bo Wang ${ }^{2}$, \\ Haozhang Huang ${ }^{2}$, Ning Tan ${ }^{1,2,3}$, Yong Liu ${ }^{1,2,3}$, Shuisheng Wei ${ }^{2}$, Jiyan Chen ${ }^{1,2,3}$
}

${ }^{1}$ Guangdong Provincial People's Hospital, School of Medicine, South China University of Technology, Guangzhou, China; ${ }^{2}$ Department of Cardiology, Guangdong Provincial Key Laboratory of Coronary Heart Disease Prevention, Guangdong Cardiovascular Institute, Guangdong Provincial People's Hospital, Guangdong Academy of Medical Sciences, Guangzhou, China; ${ }^{3}$ The Second School of Clinical Medicine, Southern Medical University, Guangzhou, China

Contributions: (I) Conception and design: G Chen, Z Huang, Y Liu, J Chen; (II) Administrative support: G Chen, L Liu, L Meng, M Li, J Liu, S Chen, N Tan, S Wei; (III) Provision of study materials or patients: All authors; (IV) Collection and assembly of data: All authors; (V) Data analysis and interpretation: G Chen, X Yan, L Meng, S Chen; (VI) Manuscript writing: All authors; (VII) Final approval of manuscript: All authors.

\#These authors contributed equally to this work.

Correspondence to: Jiyan Chen, MD, PhD, FACC, FESC. Department of Cardiology, Guangdong Provincial Key Laboratory of Coronary Disease, Guangdong Cardiovascular Institute, Guangdong Provincial People’s Hospital, South China University of Technology, Guangzhou, China. Email: chenjiyandr@126.com; Shuisheng Wei, MD. Department of Cardiology, Guangdong Cardiovascular Institute, Guangdong Provincial People's Hospital, Guangzhou, China. Email: weishuishengdr@126.com.

Background: Dialysis-requiring acute kidney injury (AKI-D) is a potentially serious complication associated with high in-hospital mortality among patients with coronary artery disease (CAD) after coronary angiography (CAG). Patients with existing advanced kidney disease (AKD) have an increased risk of developing AKI-D. However, few studies have investigated the prognosis of AKI-D in patients with both CAD and AKD.

Methods: In this observational study, $603 \mathrm{CAD}$ patients with $\mathrm{AKD}$ (estimated glomerular filtration rate, eGFR $<30 \mathrm{~mL} / \mathrm{min} / 1.73 \mathrm{~m}^{2}$ ) were enrolled. AKI-D was defined as acute or worsening renal failure requiring the initiation of renal dialysis. The primary endpoint was 90-day all-cause mortality. Kaplan-Meier and Cox regression analyses were used to assess the association of AKI-D and 90-day all-cause mortality among CAD patients complicated with AKD.

Results: Overall, among 603 CAD patients complicated with AKD, 83 patients (13.8\%) required AKI-D. Patients underwent AKI-D had a significantly higher rate of 90-day mortality than those who did not (13.3\% vs. $6.5 \%, \log$ rank $\mathrm{P}=0.028$ ), with an unadjusted hazard ratio (HR) of 1.28 [95\% confidence interval (CI): 1.02-1.61, $\mathrm{P}=0.032]$. After adjustment for cardiac and renal impairment, however, AKI-D was no longer associated with 90-day mortality (HR: 1.08, 95\% CI: 0.84-1.39, P=0.559). The attenuation analysis showed that after adjustment for cardiac and renal function, the residual effect of 90 -day mortality was as low as $30 \%$ of the unadjusted effect.

Conclusions: The incidence of AKI-D is high among patients with CAD complicated by AKD. The high 90-day mortality rate of patients undergoing AKI-D is mainly attributable to cardio-renal impairment.

Keywords: Coronary artery disease (CAD); advanced kidney disease (AKD); dialysis; prognosis; all-cause mortality

Submitted Aug 01, 2020. Accepted for publication Aug 30, 2020.

doi: $10.21037 /$ atm-20-6365

View this article at: http://dx.doi.org/10.21037/atm-20-6365 


\section{Introduction}

Dialysis-requiring acute kidney injury (AKI-D) is a serious complication of coronary angiography (CAG). AKI-D is associated with elevated risks of prolonged hospitalization, in-hospital mortality, consistent renal failure, and the occurrence of cardiovascular events (1-7). Despite an earlier study reporting that the incidence of AKI-D had shown significant annual decreases following the implementation of prevention strategies $(8,9)$, the America National Cardiovascular Data Registry (NCDR) Cath-PCI registry study indicated that in-hospital mortality was still extremely high among patients suffering from AKI-D.

Previous studies have reported that the incidence of AKI-D is remarkably higher in coronary artery disease (CAD) patients with advanced kidney disease (AKD) than in those without AKD (8). However, most trials involving patients undergoing CAG have either excluded or failed to properly investigate patients with AKD (10-13). Moreover, the relationship between AKI-D and prognosis after hospital discharge is not yet fully understood.

To address these gaps in our knowledge, this study aimed to examine the association of AKI-D with 90-day mortality among CAD patients complicated with AKD. We present the following article in accordance with the STROBE reporting checklist (available at http://dx.doi.org/10.21037/ atm-20-6365).

\section{Methods}

\section{Study design and participants}

The study cohort was derived from a retrospective cohort in a previous study that enrolled 1030 patients who underwent CAG and had AKD at Guangdong Provincial People's Hospital between January, 2008, and December, 2018 (NCT04407936). Patients who had a pre-angiography estimated glomerular filtration rate (eGFR) $<30 \mathrm{~mL} /\left(\mathrm{min} \cdot 1.73 \mathrm{~m}^{2}\right)$ along with complete pre-angiography data and available follow-up data were eligible for inclusion in this study. Patients who were not diagnosed with CAD by CAG ( $\mathrm{n}=116)$, who were lost to follow-up ( $\mathrm{n}=111)$, or who received dialysis before angiography $(\mathrm{n}=200)$ were excluded. Finally, 603 eligible patients were included in the study (Figure 1). The study protocol was approved by Guangdong Provincial People's Hospital (No. GDREC2019555H), and the study was performed in accordance with the Declaration of Helsinki (as revised in 2013). Individual consent for this retrospective analysis was waived.

\section{Procedures}

CAG or percutaneous coronary intervention (PCI) was performed according to standard clinical practice using standard guide catheters, balloon catheters, and guide wires $(14,15)$. As part of routine therapy, all patients were given an infusion of isotonic saline at a rate of $0.5-1 \mathrm{~mL} / \mathrm{kg} / \mathrm{h}$, which was initiated $12 \mathrm{~h}$ before the procedure (16). The dialysis treatment strategy was left to the discretion of the treating cardiologist according to clinical protocols based on guidelines $(17,18)$. The patients were followed up through telephone contact and interviews by medical assistants or nurses, and the follow-up data were recorded.

\section{Definition and clinical outcome}

The primary exposure variable for the current analyses was the development of AKI-D, which was defined as acute or worsening renal failure requiring renal dialysis (2). AKD was defined as eGFR $<30 \mathrm{~mL} /\left(\min \cdot 1.73 \mathrm{~m}^{2}\right)$, which was calculated using the Modification of Diet in Renal Disease (MDRD) formula (19). Congestive heart failure (CHF) was defined as New York Heart Association (NYHA) class $>2$ or Killip class $>1$. Hypertension was deemed to be definitively present if the participant was under treatment with antihypertensive medication, or had systolic blood pressure $\geq 140 \mathrm{mmHg}$ or diastolic blood pressure $\geq 90 \mathrm{mmHg}$ (20). The study's primary outcome was all-cause mortality within 90 days.

\section{Statistical analysis}

The demographic, laboratory, clinical, and procedural characteristics of the study population were summarized using descriptive statistics. Descriptive statistics were reported as the mean $(\mathrm{SD})$, median [interquartile range (IQR)], or number and percentage where appropriate. The characteristics of patients who did and patients who did not receive postoperative dialysis were compared using the unpaired Student's $t$-test, the Wilcoxon rank-sum test, or the chi-square test for normally distributed, nonnormally distributed, and categorical data, respectively. The survival analysis for all-cause mortality was performed using the Kaplan-Meier method in the two groups according to the presence of AKI-D, and the log-rank test was used to evaluate the differences in survival between the two groups.

Univariate and multivariate Cox proportional hazards models were used to investigate the associations of 


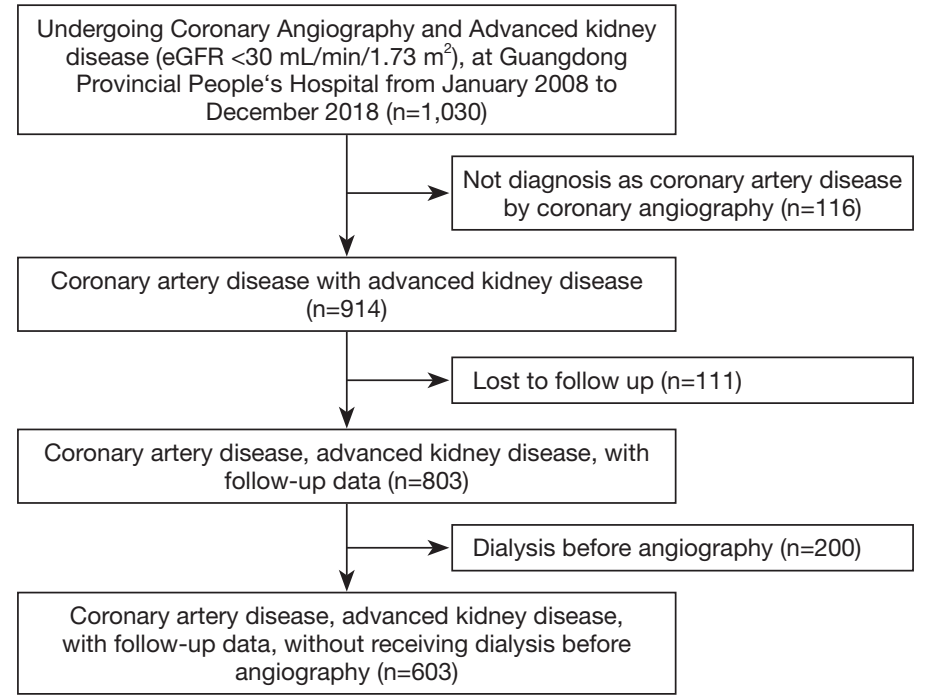

Figure 1 Flow chart for participants selection. eGFR, estimated glomerular filtration rate.

postoperative initiation dialysis with 90-day all-cause mortality. To explore the impact of postoperative initiation of dialysis on prognosis, multivariable Cox regression analyses were carried out with adjustment for the following: baseline characteristics including age, sex, diabetes mellitus, and hypertension (model 1); acute coronary syndrome (ACS) and CHF (model 2); eGFR (model 3); the combination of ACS, CHF, and eGFR (model 4). Hazard ratios (HR) and $95 \%$ confidence intervals (CIs) were calculated. Finally, the attenuation effect, which was defined as the residual effect of AKI-D on the 90-day mortality after adjustment for the 4 models were analysed as described by Kershaw et al. (21). The data were analyzed on an available case basis and missing data were not imputed.

All statistical analyses were performed using SAS (ver. 9.4) and $\mathrm{R}$ (ver. 4.0.1). A $\mathrm{P}$ values $<0.05$ was considered to represent statistical significance.

\section{Results}

This study enrolled 603 CAD patients with AKD. The baseline clinical characteristics of the patients are shown in Table 1. The patients had a mean age of $68.9 \pm 9.9$ years, and females made up $34.8 \%$ of the cohort. Comorbidities were highly prevalent among the patients and included hypertension (81.4\%), diabetes (52.6\%), and CHF (43.1\%). Almost $20.6 \%$ of patients had poor kidney function at baseline (eGFR $<15 \mathrm{~mL} / \mathrm{min} / 1.73 \mathrm{~m}^{2}$ ).

Patients who received postoperative dialysis $(n=83)$ were more likely to be younger ( 66.1 vs. 69.3 years, $\mathrm{P}=0.006)$ and less likely to be females $(24.1 \%$ vs. $36.5 \%, \mathrm{P}=0.027)$ than those not receiving postoperative dialysis $(\mathrm{n}=520)$. AKI-D patients were also more likely to have worse kidney function (eGFR $<15 \mathrm{~mL} / \mathrm{min} / 1.73 \mathrm{~m}^{2}, 54.2 \%$ vs. $15.2 \%, \mathrm{P}<0.001$ ), diabetes mellitus (62.7\% vs. 49.8\%, $\mathrm{P}=0.030)$, CHF $(62.7 \%$ vs. $40.0 \%, \mathrm{P}<0.001)$, ACS $(69.9 \%$ vs. $45.6 \%, \mathrm{P}<0.001)$ and anemia $(95.2 \%$ vs. $76.4 \%, \mathrm{P}<0.001)$. Postoperative dialysis was also associated with lower LDL-C levels (2.5 vs. $2.7 \mathrm{mmol} / \mathrm{L}, \mathrm{P}=0.013$ ) and lower rate of statins use after discharge (77.1\% vs. 92.1\%, $\mathrm{P}<0.001)$. The proportions of current smoking, hypertension, peripheral arterial disease, and the rate of $\beta$-blockers use after discharge were similar in both groups.

During the 90-day follow-up, 11 (13.3\%) and 34 (6.5\%) deaths occurred in the dialysis and non-dialysis group respectively. Kaplan-Meier survival analysis revealed a higher 90-day mortality rate in the dialysis group than in the non-dialysis group (log-rank $\mathrm{P}=0.028$; Figure 2$)$.

Univariate Cox regression analysis (Table 2) showed that AKI-D, sex, smoking, eGFR, LDL-C, white blood cell count, stains use after discharge, and $\beta$-blockers use after discharge were associated with 90 -day mortality $(\mathrm{P}<0.05)$. Figure 3 shows the unadjusted HR of AKI-D for 90-day mortality along with the HR adjusted for baseline clinical characteristics (age, sex, diabetes, and hypertension), cardiac impairment (ACS and CHF), and renal function (eGFR). Multivariate Cox regression analysis showed that after adjustment for baseline clinical characteristics, AKI-D was 
Table 1 Baseline characteristics of the study patients

\begin{tabular}{|c|c|c|c|c|c|}
\hline Characteristic* & Missing data & Overall $(\mathrm{N}=603)$ & AKI-D (N=83) & Non-AKI-D (N=520) & $P$ value \\
\hline Age, years & $0(0)$ & $68.9(9.9)$ & $66.1(9.3)$ & $69.3(10.0)$ & 0.006 \\
\hline Female, n (\%) & $0(0)$ & $210(34.8)$ & $20(24.1)$ & $190(36.5)$ & 0.027 \\
\hline \multicolumn{6}{|l|}{ Coexisting conditions, $\mathrm{n}(\%)$} \\
\hline Diabetes mellitus & $0(0)$ & $311(52.6)$ & $52(62.7)$ & $259(49.8)$ & 0.030 \\
\hline Hypertension & $0(0)$ & $491(81.4)$ & 70 (84.3) & $421(81.0)$ & 0.463 \\
\hline $\mathrm{CHF}$ & $0(0)$ & $260(43.1)$ & $52(62.7)$ & $208(40.0)$ & $<0.001$ \\
\hline Previous PCl & $0(0)$ & $114(18.9)$ & $18(21.7)$ & $96(18.5)$ & 0.486 \\
\hline Anemia, n (\%) & $2(0.3)$ & 476 (78.9.) & 79 (95.2) & $397(76.4)$ & $<0.001$ \\
\hline ACS, n (\%) & $0(0)$ & 295 (48.9) & $58(69.9)$ & $237(45.6)$ & $<0.001$ \\
\hline \multicolumn{6}{|l|}{ Estimated GFR, n (\%) } \\
\hline eGFR $<15, \mathrm{~mL} / \mathrm{min} / 1.73 \mathrm{~m}^{2}$ & $0(0)$ & $124(20.6)$ & $45(54.2)$ & $79(15.2)$ & $<0.001$ \\
\hline $15 \leq \mathrm{eGFR}<30, \mathrm{~mL} / \mathrm{min} / 1.73 \mathrm{~m}^{2}$ & $0(0)$ & $479(79.4)$ & $38(45.8)$ & $441(84.8)$ & $<0.001$ \\
\hline \multicolumn{6}{|l|}{ Laboratory examination } \\
\hline $\mathrm{HDL}-\mathrm{C}, \mathrm{mmol} / \mathrm{L}$ & $18(3.0)$ & $0.9(0.3)$ & $0.9(0.4)$ & $0.9(0.2)$ & 0.600 \\
\hline LDL-C, mmol/L & $0(0)$ & $2.7(1.0)$ & $2.5(0.9)$ & $2.7(1.0)$ & 0.013 \\
\hline
\end{tabular}

*, data are presented as the mean value standard deviation or a percentage of participants. AKI-D, dialysis-requiring acute kidney injury; $\mathrm{CHF}$, congestive heart failure; $\mathrm{PCl}$, percutaneous coronary intervention; PAD, peripheral arterial disease; eGFR, estimated glomerular filtration rate; ACS, acute coronary syndrome; HDL-C, high-density lipoprotein cholesterol; LDL-C, low-density lipoprotein cholesterol; TRIG, triglycerides; WBC, white blood cell.

still a significant risk factor for 90-day mortality (adjusted HR: 1.28, 95\% CI: 1.01-1.61, $\mathrm{P}=0.04)$. However, after adjustment for cardiac and/or renal function, dialysis was no longer an independent predictor of 90-day mortality (Table 3). Figure 4 depicts the attenuation of the relationship between dialysis and 90-day mortality that resulted from the adjustment for baseline characteristics, cardiac impairment, and/or renal function. Remarkably, after adjustment for ACS, CHF, and eGFR, the residual effect on 90-day mortality was as low as $30 \%$ of the unadjusted effect.

\section{Discussion}

To our knowledge, this study is the first to evaluate the association between AKI-D and 90-day mortality in patients with CAD complicated by AKD. The key finding of the current study is that patients who received AKI-D had a higher risk of mortality at 90 days. This association was 


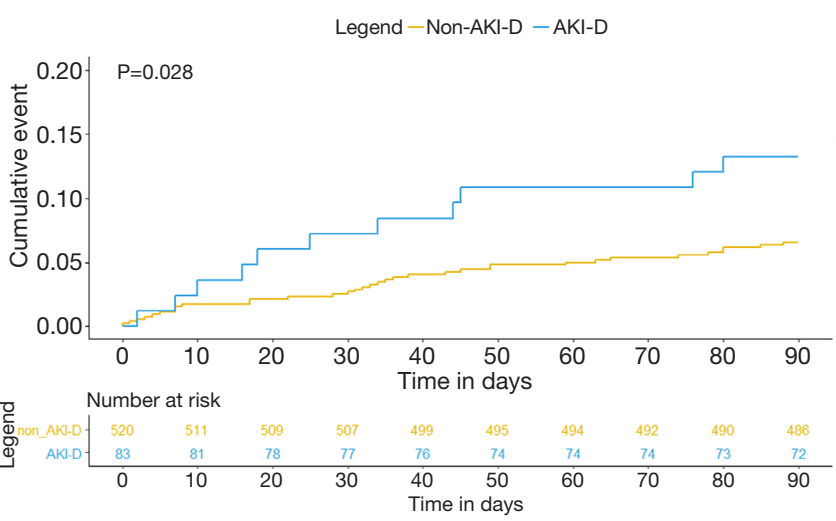

Figure 2 Kaplan-Meier curves showing cumulative hazard of 90-day mortality. AKI-D, dialysis-requiring acute kidney injury. mainly attributable to cardio-renal impairment. Indeed, after adjustment for cardiac and renal function, AKI-D was no longer an independent predictor of 90-day mortality among patients with CAD complicated by AKD.

For the incidence of AKI-D, Tsai et al.'s study investigated approximately 1 million consecutive patients who underwent PCI (between June, 2009 and June, 2011) at 1,253 sites in the USA. They estimated that the rate of AKI-D for all CAG patients was $0.3 \%$. Moreover, Brown et al. (8) reported that AKI-D affected $0.25 \%$ of the 3 million cardiac catheterization or PCI patients in the United States from 2001 to 2011. Our study included 603 very high-risk patients with $\mathrm{AKD}$ who underwent CAG or PCI between 2008 and 2018, meaning the majority of

Table 2 Univariate Cox regression analyses with risk factors for a death event at 90 days in CAD patients complicated with AKD

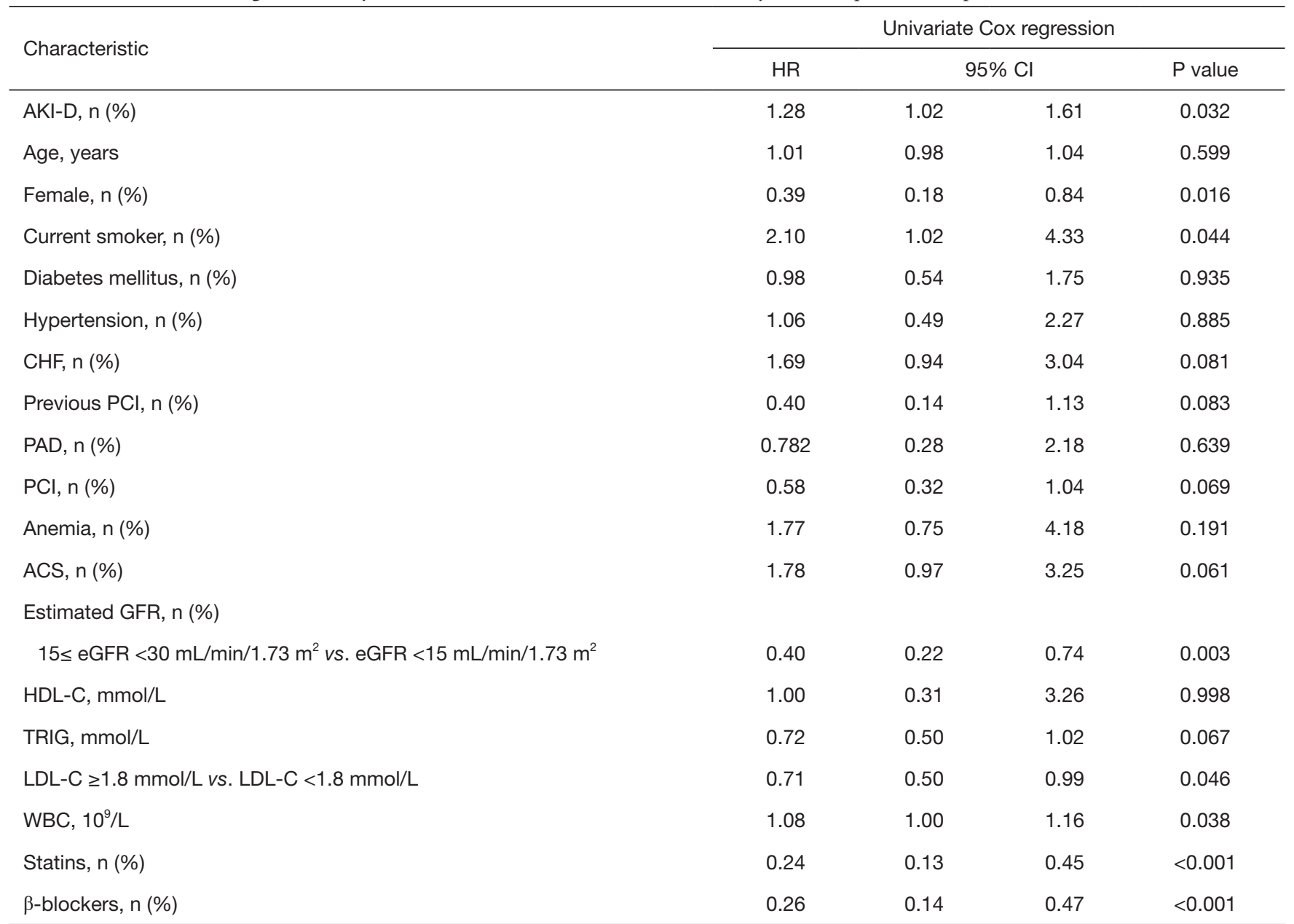

CAD, coronary artery disease; AKD, advanced kidney disease; HR, hazard ratio; Cl, confidence interval; AKI-D, dialysis-requiring acute kidney injury; CHF, congestive heart failure; PCl, percutaneous coronary intervention; PAD, peripheral arterial disease; eGFR, estimated glomerular filtration rate; ACS, acute coronary syndrome; HDL-C, high-density lipoprotein cholesterol; TRIG, triglycerides; LDL-C, lowdensity lipoprotein cholesterol; WBC, white blood cell. 


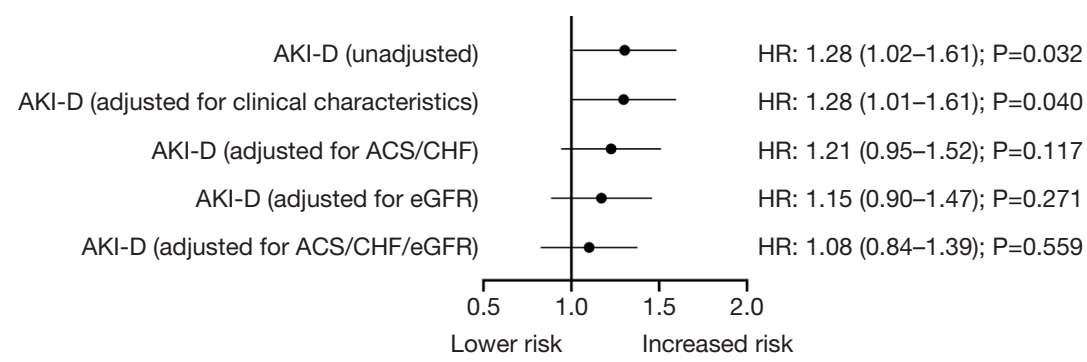

Figure 3 Unadjusted and adjusted HRs and 95\% CIs for 90-day mortality. HRs were adjusted for baseline clinical characteristics (age, sex, diabetes mellitus, and hypertension), for ACS and CHF, for eGFR, and for the combination of ACS, CHF, and eGFR. HR, hazard ratio; CI, confidence interval; AKI-D, dialysis-requiring acute kidney injury; ACS, acute coronary syndrome; CHF, congestive heart failure; eGFR, estimated glomerular filtration rate, eGFR $<15 \mathrm{~mL} / \mathrm{min} / 1.73 \mathrm{~m}^{2} v s .15 \leq \mathrm{eGFR}<30 \mathrm{~mL} / \mathrm{min} / 1.73 \mathrm{~m}^{2}$.

Table 3 Multivariate Cox regression analyses with risk factors for all-cause mortality event at 90 days in CAD patients complicated with $\mathrm{AKD}$

\begin{tabular}{|c|c|c|c|c|}
\hline \multirow{2}{*}{ Characteristic } & \multicolumn{4}{|c|}{ Multivariate Cox regression } \\
\hline & $\mathrm{HR}$ & \multicolumn{2}{|c|}{$95 \% \mathrm{Cl}$} & $P$ value \\
\hline \multicolumn{5}{|l|}{ Model 1} \\
\hline AKI-D, n (\%) & 1.28 & 1.01 & 1.61 & 0.040 \\
\hline Age, years & 1.02 & 0.99 & 1.05 & 0.198 \\
\hline Female, n (\%) & 0.38 & 0.17 & 0.82 & 0.014 \\
\hline Diabetes mellitus, $\mathrm{n}(\%)$ & 1.02 & 0.56 & 1.87 & 0.938 \\
\hline Hypertension, n (\%) & 1.00 & 0.46 & 2.17 & 0.995 \\
\hline \multicolumn{5}{|l|}{ Model 2} \\
\hline AKI-D, n (\%) & 1.21 & 0.95 & 1.52 & 0.117 \\
\hline ACS, n (\%) & 1.59 & 0.86 & 2.93 & 0.142 \\
\hline CHF, n (\%) & 1.51 & 0.83 & 2.75 & 0.175 \\
\hline \multicolumn{5}{|l|}{ Model 3} \\
\hline AKI-D, n (\%) & 1.15 & 0.90 & 1.47 & 0.271 \\
\hline eGFR ${ }^{\#}$ & 0.46 & 0.24 & 0.88 & 0.020 \\
\hline \multicolumn{5}{|l|}{ Model 4} \\
\hline AKI-D, n (\%) & 1.08 & 0.84 & 1.39 & 0.559 \\
\hline ACS, n (\%) & 1.64 & 0.89 & 3.03 & 0.116 \\
\hline CHF, n (\%) & 1.60 & 0.88 & 2.92 & 0.123 \\
\hline eGFR ${ }^{\#}$ & 0.95 & 0.91 & 0.99 & 0.025 \\
\hline
\end{tabular}

\#, eGFR, eGFR $<15 \mathrm{~mL} / \mathrm{min} / 1.73 \mathrm{~m}^{2}$ vs. $15 \leq$ eGFR $<30 \mathrm{~mL} / \mathrm{min} / 1.73 \mathrm{~m}^{2}$. CAD, coronary artery disease; AKD, advanced kidney disease; HR, hazard ratio; $\mathrm{Cl}$, confidence interval; AKI-D, dialysis-requiring acute kidney injury; ACS, acute coronary syndrome; CHF, congestive heart failure; eGFR, estimated glomerular filtration rate. patients included in this study were treated according to the latest clinical guidelines. In our study, the incidence of AKI-D in AKD patients who underwent CAG was $13.8 \%$. The reason why the incidence in our study was much higher than those of other studies is that we included the very high risk patients with comorbidity of both $\mathrm{CAD}$ and $\mathrm{AKD}$. Therefore, due to the high incidence of AKI-D among AKD patients, the prognosis of AKD patients who receive AKI-D after CAG would be worthy of further investigation.

The mortality rate is considerably greater in patients with CAD who present with AKI-D than in those without AKI-D, despite improvements to therapeutic strategies $(8,9)$. In the intervention era, Brown et al. and Tsai et al. reported in-hospital mortality rates for AKI-D of $20 \%$ and $34 \%$, respectively. However, the 90-day mortality rate for patients in our study was $13.3 \%$, which is slightly lower than those in the studies of Brown and Tsai. The main reasons for these differences are as follows. First, the dialysis rate in the AKD patients in our study (13.8\%) was much higher than that in Tsai's study (4.20\%), which may be explained by their study having more stringent indications for dialysis. Essentially, the AKI-D patients included in Tsai's study may have been at a higher risk than our patients. Second, the clinical data collected by Brown et al. and Tsai et al. were both before 2011, while our study was conducted between 2008 and 2018, during which time, the treatment for critically ill patients has been greatly improved. Moreover, Weisbord et al. (1) found that CI-AKI was associated with a 4 -fold increase in relative risk for 90-day death, need for dialysis, and persistent kidney impairment in 4,418 participants after adjusting for age, sex, urine albuminto-creatinine ratio, diabetes, and myocardial infarction. The results of our study indicated that AKI-D was associated with an increased risk for 90-day mortality after adjusting 


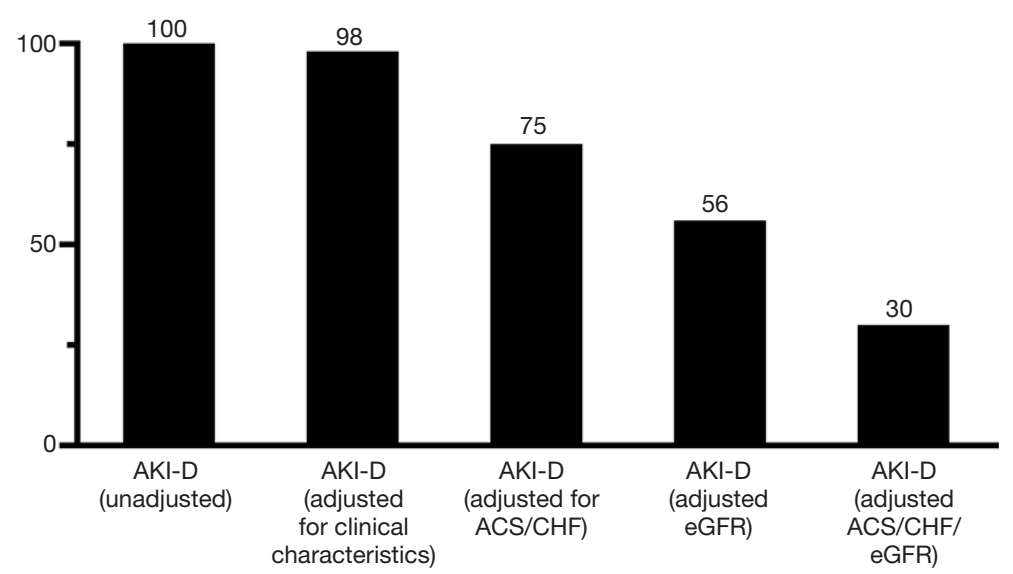

Figure 4 Residual effect (\%) of AKI-D on 90-day mortality. AKI-D, dialysis-requiring acute kidney injury; ACS, acute coronary syndrome; CHF, congestive heart failure; eGFR, estimated glomerular filtration rate, eGFR $<15 \mathrm{~mL} / \mathrm{min} / 1.73 \mathrm{~m}^{2} v s .15 \leq \mathrm{eGFR}<30 \mathrm{~mL} / \mathrm{min} / 1.73 \mathrm{~m}$.

for age, sex, DM and hypertension (HR: 1.275, 95\% CI: 1.011-1.607). Weisbord et al. chose composite events as the primary outcomes of their study, which may have led to the risk of CI-AKI being overestimated. In contrast, we chose all-cause mortality as the clinical outcome of our investigation, which is considered to be the most significant outcome for patient prognosis. Furthermore, our study included AKD patients with CAG; these patients are usually considered to be a population at very high risk and with a poor prognosis (22-25).

Our analysis indicated that AKI-D might be associated with a poorer prognosis in patients with CAD complicated by AKD. However, the prognostic power of AKI-D for 90-day mortality lost significance after adjustment for cardiac and renal function. These results were unsurprising. Previous studies have indicated that cardiac function and renal function are among the most significant predictive factors for out-of-hospital mortality $(26,27)$. Moreover, clinicians' judgements of postoperative dialysis also mainly depend on patients' cardiac and renal function. Therefore, our results support the well-known opinion that a dialysis requirement is associated with poor prognosis in patients with CAD complicated by AKD. More importantly, we demonstrated that the poorer outcome may largely be explained by cardiac and renal dysfunction.

Our data may provide a better understanding of the prognosis for AKD patients who receive CAG. First, our analysis, which indicated that the incidence of AKI-D among CAD patients complicated with AKD was $13.8 \%$, was conducted from 2008 to 2018, meaning our results are more likely to reflect current dialysis rates under the latest clinical guidelines (16). Second, while the association between AKI-D and the prognosis of AKD patients is unclear, our study found that the risk of 90-day mortality in AKD patients with AKI-D was 1.28 times higher than that in AKD patients without AKI-D, which may fill this gap and contribute to raising awareness of the need to preserve kidney function in such high-risk patients. Attention should be given to discharge management, as well as to in-hospital prevention or treatment, such as intravascular volume expansion, prophylactic haemofiltration and so on. Third, our analysis indicated that the increased 90-day mortality of patients undergoing AKI-D is mainly due to cardiorenal impairment, which suggests that cardio- and renalprotective effects should be the focus of future studies on CAD patients complicated with AKD.

Our study has several limitations. First, the study was a single-center, retrospective study, which may lead to the occurrence of selective bias, limiting the usefulness of these results. In order to avoid the bias, all eligible consecutive patients within the designated timeframe were included and all measurements were taken while blinded to the patient outcome. Second, only CAD patients complicated with advanced CKD, a relatively undiversified population, were included. However, this also means that our research is more targeted, and the prognosis of these patients has not been studied before. Finally, although our multivariable models were adjusted for some prognostic predictors, there is a possibility that the results were affected by unmeasured confounders, such as occurrence of tumor, medication compliance and the intensity of physical exercise.

In conclusion, the incidence of AKI-D is high in patients with CAD complicated by AKD. In our study, AKI-D was significantly associated with an increased relative risk of 
90-day mortality, which was mainly associated with cardiorenal impairment. Clinicians should afford more attention to discharge management in these high-risk patients.

\section{Acknowledgments}

Funding: This study was supported by the National Science Foundation of China (Grant No. 81670339 and Grant No. 81970311), Cardiovascular Research Foundation Project of the Chinese Medical Doctor Association (SCRFCMDA201216), the Progress of Science and Technology Project in Guangdong Province (2017B020247060) and Beijing Lisheng Cardiovascular Health Foundation (LHJJ20141751 and LHJJ201612127). YL receives research funding from National Science Foundation of China (Grant No. 81970311). JC receives research funding from National Science Foundation of China (Grant No. 81670339), Cardiovascular Research Foundation Project of the Chinese Medical Doctor Association (SCRFCMDA201216) and Beijing Lisheng Cardiovascular Health Foundation (LHJJ20141751). JL receives research funding from the Progress of Science and Technology Project in Guangdong Province (2017B020247060) and Beijing Lisheng Cardiovascular Health Foundation (LHJJ201612127). The funders had no role in the study design, data collection and analysis, decision to publish, or preparation of the manuscript; the work was not funded by any industry sponsors.

\section{Footnote}

Reporting Checklist: The authors have completed the STROBE reporting checklist. Available at http://dx.doi. org/10.21037/atm-20-6365

Data Sharing Statement: Available at http://dx.doi. org/10.21037/atm-20-6365

Conflicts of Interest: All authors have completed the ICMJE uniform disclosure form (available at http://dx.doi. org/10.21037/atm-20-6365). The authors have no conflicts of interest to declare.

Ethical Statement: The authors are accountable for all aspects of the work in ensuring that questions related to the accuracy or integrity of any part of the work are appropriately investigated and resolved. The study protocol was approved by Guangdong Provincial People's
Hospital (No. GDREC2019555H), and the study was performed in accordance with the Declaration of Helsinki (as revised in 2013). Individual consent for this retrospective analysis was waived.

Open Access Statement: This is an Open Access article distributed in accordance with the Creative Commons Attribution-NonCommercial-NoDerivs 4.0 International License (CC BY-NC-ND 4.0), which permits the noncommercial replication and distribution of the article with the strict proviso that no changes or edits are made and the original work is properly cited (including links to both the formal publication through the relevant DOI and the license). See: https://creativecommons.org/licenses/by-nc-nd/4.0/.

\section{References}

1. Weisbord SD, Palevsky PM, Kaufman JS, et al. ContrastAssociated Acute Kidney Injury and Serious Adverse Outcomes Following Angiography. J Am Coll Cardiol 2020;75:1311-20.

2. Tsai TT, Patel UD, Chang TI, et al. Contemporary Incidence, Predictors, and Outcomes of Acute Kidney Injury in Patients Undergoing Percutaneous Coronary Interventions. JACC: Cardiovascular Interventions 2014;7:1-9.

3. Liangos O, Wald R, O'Bell JW, et al. Epidemiology and outcomes of acute renal failure in hospitalized patients: a national survey. Clin J Am Soc Nephrol 2006;1:43-51.

4. Hsu CY, Chertow GM, McCulloch CE, et al. Nonrecovery of kidney function and death after acute on chronic renal failure. Clin J Am Soc Nephrol 2009;4:891-8.

5. Metnitz P, Krenn C, Steltzer H, et al. Effect of acute renal failure requiring renal replacement therapy on outcome in critically ill patients. Critical care medicine 2002;30:2051-8.

6. Lo LJ, Go AS, Chertow GM, et al. Dialysis-requiring acute renal failure increases the risk of progressive chronic kidney disease. Kidney Int 2009;76:893-9.

7. Wu VC, Shiao CC, Chang CH, et al. Long-term outcomes after dialysis-requiring acute kidney injury. Biomed Res Int 2014;2014:365186.

8. Brown JR, Rezaee ME, Nichols EL, et al. Incidence and In-Hospital Mortality of Acute Kidney Injury (AKI) and Dialysis-Requiring AKI (AKI-D) After Cardiac Catheterization in the National Inpatient Sample. J Am Heart Assoc 2016;5:e02739.

9. Lenihan CR, Montez-Rath ME, Mora Mangano CT, et 
al. Trends in acute kidney injury, associated use of dialysis, and mortality after cardiac surgery, 1999 to 2008. Ann Thorac Surg 2013;95:20-8.

10. Nijssen EC, Rennenberg RJ, Nelemans PJ, et al. Prophylactic hydration to protect renal function from intravascular iodinated contrast material in patients at high risk of contrast-induced nephropathy (AMACING): a prospective, randomised, phase 3, controlled, open-label, non-inferiority trial. Lancet 2017;389:1312-22.

11. Weisbord SD, Gallagher M, Jneid H, et al. Outcomes after Angiography with Sodium Bicarbonate and Acetylcysteine. N Engl J Med 2018;378:603-14.

12. Maioli M, Toso A, Leoncini M, et al. BioimpedanceGuided Hydration for the Prevention of ContrastInduced Kidney Injury. Journal of the American College of Cardiology 2018;71:2880-9.

13. Narula A, Mehran R, Weisz G, et al. Contrast-induced acute kidney injury after primary percutaneous coronary intervention: results from the HORIZONS-AMI substudy. European Heart Journal 2014;35:1533-40.

14. Kushner FG, Hand M, Smith SC, Jr., et al. 2009 focused updates: ACC/AHA guidelines for the management of patients with ST-elevation myocardial infarction (updating the 2004 guideline and 2007 focused update) and ACC/AHA/SCAI guidelines on percutaneous coronary intervention (updating the 2005 guideline and 2007 focused update) a report of the American College of Cardiology Foundation/American Heart Association Task Force on Practice Guidelines. J Am Coll Cardiol 2009;54:2205-41.

15. Jneid H, Anderson JL, Wright RS, et al. 2012 ACCF/AHA focused update of the guideline for the management of patients with unstable angina/non-ST-elevation myocardial infarction (updating the 2007 guideline and replacing the 2011 focused update): a report of the American College of Cardiology Foundation/American Heart Association Task Force on Practice Guidelines. J Am Coll Cardiol 2012;60:645-81.

16. Neumann FJ, Sousa-Uva M, Ahlsson A, et al. 2018 ESC/ EACTS Guidelines on myocardial revascularization. Eur Heart J 2019;40:87-165.

17. Tattersall J, Dekker F, Heimburger O, et al. When to start dialysis: updated guidance following publication of the Initiating Dialysis Early and Late (IDEAL) study. Nephrol Dial Transplant 2011;26:2082-6.

18. Stevens PE, Levin A. Evaluation and management of chronic kidney disease: synopsis of the kidney disease: improving global outcomes 2012 clinical practice guideline. Ann Intern Med 2013;158:825-30.

19. Aguiar-Souto P, Ferrante G, Del Furia F, et al. Frequency and predictors of contrast-induced nephropathy after angioplasty for chronic total occlusions. 2010;139:68-74.

20. Wilson P, D'Agostino R, Levy D, et al. Prediction of coronary heart disease using risk factor categories. 1998;97:1837-47.

21. Kershaw KN, Droomers M, Robinson WR, et al. Quantifying the contributions of behavioral and biological risk factors to socioeconomic disparities in coronary heart disease incidence: the MORGEN study. Eur J Epidemiol 2013;28:807-14.

22. Gharaibeh KA, Hamadah AM, Sierra RJ, et al. The Rate of Acute Kidney Injury After Total Hip Arthroplasty Is Low but Increases Significantly in Patients with Specific Comorbidities. J Bone Joint Surg Am 2017;99:1819-26.

23. Hassan BK, Sahlstrom A, Dessau RB. Risk factors for renal dysfunction after total hip joint replacement; a retrospective cohort study. J Orthop Surg Res 2015;10:158.

24. Jafari SM, Huang R, Joshi A, et al. Renal impairment following total joint arthroplasty: who is at risk? J Arthroplasty 2010;25:49-53, e1-2.

25. Wu KT, Chen CY, Chen B, et al. The Incidence and Risk Factors of Acute Kidney Disease after Total Knee Arthroplasty with Early Postoperative Volume Supplement. Biomed Res Int 2018;2018:8718545.

26. Engelbertz C, Reinecke H, Breithardt G, et al. Two-year outcome and risk factors for mortality in patients with coronary artery disease and renal failure: The prospective, observational CAD-REF Registry. Int J Cardiol 2017;243:65-72.

27. Vidal-Petiot E, Greenlaw N, Kalra PR, et al. Chronic Kidney Disease Has a Graded Association with Death and Cardiovascular Outcomes in Stable Coronary Artery Disease: An Analysis of 21,911 Patients from the CLARIFY Registry. Journal of Clinical Medicine 2019;9:4.

(English Language Editor: J. Reynolds)

Cite this article as: Chen G, Yan X, Huang Z, Liu L, Meng L, Li M, Liu J, Chen S, Li H, Mai Z, Chen E, Lai D, Wang B, Huang H, Tan N, Liu Y, Wei S, Chen J. Association of dialysisrequiring acute kidney injury with 90-day prognosis in patients with coronary artery disease and advanced kidney disease after coronary angiography. Ann Transl Med 2020;8(19):1241. doi: 10.21037/atm-20-6365 